Ophthalmologica 1961;142(suppl. 1):I-III

\title{
Contents, Vol. 142, Supplement 1, 1961
}

\section{Contents}

Introduction

Data for the Present Analysis

Predictable Unity Correlations

Partial Correlations

Multiple Correlations
4

12

Partial and Multiple Correlation Coefficients for Constant Refraction

Multiple Regressions 19

The Relation between Total Refractive Power and Axial Length . . 20

Analysis of Simple Correlations 25

Diagrammatic Representation 25

Common Factors 29

Factor Analysis . 31

The Nature of the Factor S

The Nature of the Factor $P$

The Movement of the Choroid on Parasympathetic and Sympathetic Stimulation 44

The Pressure in the Suprachoroidal Space

The Pressure on the Sclera

49

The Tension in the Choroid (Direct Recording)

Effect of Autonomic Drugs on Isolated Ciliary Muscle-Choroid Preparations

The Origin of Emmetropia and Ametropia 58

The Shape of the Eye and the Nature of the Factor $\mathrm{R}$

Higher Centers 63

Psychological Factors $\quad 65$

School Myopia 68

The Relation Between the Distributions of the Optical Elements ... 69

Appendix: A Note on Presbyopia and Glaucoma

Presbyopia

74

Narrow Angle Glaucoma

Wide Angle Glaucoma

D. Discussion

79

Summary

88 